\title{
Neurological Foundation of Unconscious and Higher Thinking Processes and the Dynamic Variability of Clinical Presentations
}

\author{
Lawrence Greenman \\ Hudson Valley Health Care System, Poughkeepsie, NY, USA \\ Email: lgreenmanmd@msn.com
}

How to cite this paper: Greenman, L. (2018) Neurological Foundation of Unconscious and Higher Thinking Processes and the Dynamic Variability of Clinical Presentations. Open Journal of Psychiatry, 8, 73-79.

https://doi.org/10.4236/ojpsych.2018.81006

Received: December 1, 2017

Accepted: January 9, 2018

Published: January 12, 2018

Copyright $\odot 2018$ by author and Scientific Research Publishing Inc. This work is licensed under the Creative Commons Attribution International License (CC BY 4.0).

http://creativecommons.org/licenses/by/4.0/

\begin{abstract}
This article reviews selected neuroscientific and psychoanalytic writings with a focus on dynamic variability, unconscious processes and their presence in clinical venues. Two objects are pursued. First is the development of neuron circuity, leading up to higher thought processes and thinking. Second is to elucidate the neuron circuitry of unconscious processes. The goal is to describe the stated objectives and the theme of variability represented in clinical presentations and venues.
\end{abstract}

\section{Keywords}

Genome, Neurodevelopment, Dynamic Variability, Clinical Venues

\section{Introduction}

The entirety of an organism's hereditary information is contained in the genome. Constituents of the genome are represented on receptor mechanisms of the cells of the central nervous system (CNS), autonomic nervous system (ANS), and the peripheral nervous system (PNS). Elemental potential for variability lies within the gene itself. According to the Encode Project [1], it is not just the gene but its network that makes the gene dynamic ... transcription factors-special genes that can simultaneously activate or silence thousands of genes that are wired together in hierarchal fashion ... Kandal [2] states that, "genes and proteins determine neural connections and experience, including psychotherapy, alters gene expression ... network diagrams of hierarchical organization in the brain have given way to dynamic models of neuronal activity, involving abundant recursive connections between the brain regions and subtle temporal and state changes that have been hypothesized to underlie mental function. Information carried by 
a gene is defined by an assembly of a protein chain (in the double helix). The sequence of amino acids in a protein determines how the chain folds and therefore how it assumes the three-dimensional structure necessary for biological activity. Internal and external stimulation steps in the development of the brain, hormones, stress, learning, and social interactions alter aspects of gene regulation ... This is sometimes referred to epigenetic gene regulation". It is fundamental to keep in mind that a cardinal principal of gene expression is represented by the cell membranes of the all organ systems. It is of equal significant to recognize that all the foregoing is modulated by the sense organs and includes the development of the archaic mind, the foundation for unconscious process. Kandal argues that, "In the case of consciousness, it is hard to imagine any solution that would not also require an understanding of the large neural networks that underlies cognition, actions and emotions". In a commentary on Kandal's contributions, Hyman [3] states "The implication for psychiatry in Kandal's work and that of others who have worked on brain plasticity is that life experience and indeed all types of learning, including psychotherapy, influence thinking, emotion, and behavior by modifying synaptic connections in certain brain circuits. Moreover, as scientists have shown, circuits are shaped over lifetime by multiple complexly interacting factors including genes, illness injury, experience, context and chance". Hymen [4] describes the neurobiology of clinical addictions which he believes might serve as a model for the plasticity of the brain regarding emotional pleasure factors. His model involves the transcription of messenger ribonucleic acid over a period of several months. Edelman [5] states that in neurodevelopment, "Neurons extend the branching process in many directions, generating extensive variability in the connective patterns of neural circuits. Neurons that fire together wire together in the developing individual which persists throughout each individual's lifetime, include those circuits linked to emotional, affective centers in the brain". These links are widely believed to be involved with evolutionary imperatives, so-called instinctual drives related to survival (aggressive) and propagation (sexual) of the human species.

Unique for the human species is the acquisition of speech and language. As it develops, groups of neurons involved in the communications are set down within the nervous system including the archaic mind. I would like to underline that neurons develop within the nervous system simultaneously with each and every organ system of the body which provides input to it in response to complex internal and external events channeled by the sense organs. Scientists have referred to the human mind's capacity to use past and present information to plan for and adapt to the possible future. The pathway to this type of thinking is, for the most part, the result of speech and language. It allows for the open-ended formation of concepts. It pertains to a capacity for metaphor, symbolism, and abstraction.

The functional process of thinking was described by Einstein [6] "What precisely is thinking?" When on the reception of sense impressions, memory of such pictures form sequences, each of which calls forth another, this too is not 
yet "thinking" When, however, a picture turns up in many sequences, then precisely by such return, it becomes an organizing element for such sequences in that it connects sequences in themselves unrelated to each other. Such an element becomes a tool, a concept. I think the transition from free association or "dreaming" to thinking is characterized by the more or less pre-eminent role played by the "concept". It is by no means necessary that a concept be led to a sensory cognizable and reproducible sign (word); but when this is the case, then thinking becomes thereby capable of being communicated ... "I have no doubt that our thinking goes on for the most part without the use of signs (words) and beyond that to a considerable degree unconsciously". Although Einstein was discussing conscious thinking, he was clearly cognizant of the unconscious determinants of the thinking processes and stated that he was thereby able to recognize the failure of the absolute character of time, or that simultaneity was rooted in the unconscious. Einstein was referring to an astronomical context whereby what one sees is not necessarily happening at the time one sees it. He stated that this recognition led him to his special theory of relativity and subsequently to his general theory of relativity. In psychiatry, the neurosciences, and the larger field of mental health a theory is needed capture the range of human behavior and psychological parameters. In his autobiography, Einstein [6] defined his criteria for a serviceable theory: "A theory is more impressive, the greater the simplicity of its premises, the more kinds of things it relates, and the more extended the area of its applicability". A workable theory, therefore, encompasses and accounts for the widest range of human behavior, normal and abnormal. I believe that Freud's structural theory fits Einstein's criteria for a valid theory. The id ego, and super ego are fundamental concepts of the mental apparatus which copes with the biologically based libidinal and aggressive drives in unconscious and conscious dimensions. Application of the structural theory has accounted for the widest range of human phenomena encompassing feelings, conflict of emotions and the thinking processes intertwined with them.

Unique for the human species is that it develops in conjunction with motor, sensory, and mental interplay, including fantasy. The long period of childhood dependency on parents/caregivers necessarily incorporates those figures in the neuropsychological development of individuals. Damasio [7] states, "emotions also effect the modes of operation of numerous brain circuits; the variety of emotional responses is both responsible for profound changes in both the body landscape and the brain landscape". Brenner [8] maintains that the mind is in continuous intrinsic conflict and attempts to resolve conflicts by way of compromise formation in thought and somatic action. He argues that thought, perception, and mental representations have unconscious and conscious participation. I would add that thought and action are grounded in neurological networks via intercellular and trans-organ communication. The connecting mechanisms are propagated by the candidate gene receptors in nerve cell membranes of the CNS, ANS, and PNS. According to the Structural Theory, unconscious and conscious processes are merged in varying proportions which determine the outcomes of thoughts, feelings, emo- 
tions, and actions. Neural networks have pathways to emotional centers located in the mid brain with projection to the cerebral cortex.

At this point one might ask what exactly is the neuron circuitry of unconscious processes? The answer is that it is embedded in the CNS, ANS, and PNS in synchronization with the archaic mind. A repository of the archaic mind remains and continues to react to internal stimuli and interacts with the external world modulated by the cognitive senses, and memory. Both the Structural Theory and unconscious processes are functional dynamic entities which drive the neuropsychological processes. Mind and body are one and the same.

Fenichel [9] stated, "Posthypnotic suggestion demonstrates the existence of a psychic unconscious before our very eyes. The forgetting of a name makes us feel it subjectively. One knows that one knows the name and still one does not know it". Freud distinguished between primary process thinking and primary process thinking which has relevance today-for example, in the formation of delusions. Kandal [10] states, "Because primary process is freer and hyper associative, it is thought to facilitate the emergence of creativity that promotes new combinations and permutation of ideas-that is equivalent to an Aha! Moment where the full focus of secondary process thinking is required for the working through-the elaboration of creative insight...like much of our cognitive and affective life, even our decision making is partially unconscious suggests that unconscious mental processes are necessary for creative thinking as well".

Psychopharmacology has targeted receptor sites with complex affinities and different biogenic amines. The specifics of the nervous systems do not necessarily carry over to clinical reality. Double blind studies comparing a drug to placebo or another drug of similar type have protocols with strict exclusion criteria to attain a resemblance of the comparison groups of patients. Unfortunately, the protocols exclude the vast majority of patients treated in hospitals and clinics. Leucht, [11] et al. reviewed sixty years of placebo-controlled antipsychotic drug trails in acute schizophrenia using meta-analysis and meta-regression of efficacy predictors. It was concluded that, "Clinicians can expect that approximately two times more patients improve when treated with antipsychotics compared with placebo, but only a minority will experience a good response in the short term". Kotov, [12] et al. described the clinical course of psychotic disorders over the two decades following first hospitalization. The recruitment of patients was between 1990-1995.

A total of 373 patients completed the 20-year follow-up; 175 had schizophrenia/ schizoaffective disorder (47\%). The authors concluded that, "a high symptom burden in psychotic disorders that increases with time and ultimately may undo initial treatment gains".

Neuro-stimulation approaches are undergoing trials to treat refractory depression. Long standing electroconvulsive therapy featured a general disruption of nerve/brain impulses. In current times a more targeted approach is used such as repetitive transcranial magnetic stimulation, transcranial direct current stimulation, vagal nerve stimulation, and deep brain stimulation. Each one seeking to tem- 
porarily relieve refractory mental disorders by disrupting the functional flow of nerve impulses in the brain. These somatic techniques may eventually find their way as treatment modalities. There is no shortage of residual psychopathology begging for relief. Decades of research for biological markers have been disappointing. There is, to date, no valid biological test to diagnose a single psychiatric disorder of the major functional types met in clinics. That is, for Bipolar, Schizophrenia, Depressive Disorders, or Anxiety Disorders. The struggle for treating psychiatric illness is destined to be ongoing for the foreseeable future. The concept of neurodevelopment including the functional entities of unconscious processes and the structural theory embedded within the neuro systems by ways, cross ways, projections, and trans-organ highways is in accordance with the theme of variability.

\section{Dynamic Variability}

A perusal of the DIAGNOSTIC AND STATISTICAL MANUAL OF MENTAL DISORDERS, DSM-V [13] endorses the theme of variability. Its authors strive for diagnostic specificity in some way to match the specifics of neuroscience with multiple criteria, specifiers, modifiers and even unspecified sub diagnostic criteria. It thereby demonstrates the variability of clinical presentations. Special cautions about diagnosis are indicated in the event of involvement with substance abuse and/or medical illnesses. In order to have a working knowledge for the management of patients a grasp of basic neurodevelopmental and psychological factors would be useful. The current editions are based on behavior and clinical symptoms which has its practical advantages, but it essentially leaves out the etiologies of mental illnesses. For the latter, an understanding of human behavior and the dynamic variability of unconscious processes needs to be taken into account whenever possible. It is grounded after all in neuroscience. This perspective will have application across the wide array of clinical venues.

Of particular importance is the dynamic variability of unconscious mechanisms since it involves all mentally disordered individuals as well as all members of the treatment teams. Each has gone through more or less the same neurological wiring and has grown up under culturally similar circumstances. There are ample common grievances in the growing up processes. Arlow [14] states, "The intrusion of unconscious fantasy thinking into conscious mental experience is well known to us from the study of hallucinations, dreams, and daydreams. More recent investigation has been shown how this tendency plays a role in the structuring of perception and in the interpretation of external reality". Clinicians through their respective discipline training will presumably have more control of the factors of similar variabilities and use their knowledge to the advantage of the client/patient. It is clinicians and staff who treat patients not programs or protocols. Every routine psychiatric assessment contains an individual history of a disorder and a mental status examination. The clinical interview is based on manifestation and coordination between the CNS, ANS, PNS, and the functional men- 
tal apparatus. To illustrate: The organization of verbal associations, affect (emotions), psychomotor activity, vegetative functions (sleep, appetite), thought content (delusions, hallucinations), and behavior in different situations are described. All the clinical variables are considered in arriving at a diagnosis that appears in DSM-V. Clinical diagnosis is the basis of treatment planning.

\section{Clinical Venues}

The wide spectrum of clinical venues is in keeping with the theme of variability, and levels of care and treatment. In-patient, out-patient, incarcerated patients, and community patients. There are auxiliary programs, partial hospital programs, diversion programs, rehabilitation programs, holding units, stabilization units, crisis teams, and out-reach teams. At times the programs are conceived by clinical administrators far removed from the clinical realities of the settings. It is not uncommon for patients to be transferred from one setting to another in the acute and chronic stages of their illness. The subjects are referred to differently in different venues as patients, clients, customers, or guests.

The long period of dependency of humans on their parents/caregivers sets the stage for regression which is ubiquitous in mental life. Given the general chronicity of mental illness for many patients, there is a tendency for it to occur. Society responds to this dynamic by tilting toward providing the basic necessities of life-food, clothing, and shelter.

\section{Conclusion}

Neuroscience has provided a foundation for neurodevelopment from the hereditary genome up through consciousness. The human species singular development of speech and language has enabled a pathway to higher thinking. Neurodevelopment is described here as a coordination between the CNS, ANS, PNS, and the archaic mind consisting initially as a matrix of instinctual drives. The Structural Theory derived from neurodevelopment is the most comprehensive model to understand human behavior. Both the Structural Theory and unconscious processes are functional entities embedded in the neuron circuitry of the CNS, ANS, PNS, and the archaic mind. The latter becomes differentiated via sense organs from the internal and external world. Unconscious processes are characterized by dynamic variability. Clinical variability is the general rule in clinical presentations. Clinicians and society provide clinical venues for the care and treatment of mentally disordered patients. An operational understanding of neurodevelopment and psychoanalytic findings can be of benefit to those who labor in the field of mental health.

\section{Acknowledgements}

The author would like to thank Robert and Jax Kovacs for their assistance in the preparation of the manuscript. 


\section{References}

[1] Yale University (2012) Yale Team Finds Order Amidst the Chaos within the Human Genome. Science Daily.

[2] Kandel, E.R. (2005) Psychiatry, Psychoanalysis, and the New Biology of the Mind. American Psychiatric Publishing Inc., Arlington, 28-42.

[3] Hymen, S.E. (2005) Psychiatry, Psychoanalysis, and the New Biology of the Mind. American Psychiatric Publishing Inc., Arlington.

[4] Hymen, S.E. and Nestler, E.J. (1996) Initiation and Adaption: A Paradigm for Understanding Psychotropic Drug Reaction. American Journal of Psychiatry, 153, 151-162. https://doi.org/10.1176/ajp.153.2.151

[5] Edelman, G.M. and Tonino, G. (2000) A Universe of Consciousness: How Matter Becomes Imagination. Basic Books, New York, 79-92.

[6] Einstein, A. and Schlipp, P. (1999) Autobiographical Notes. Open Court Publishing Co., Peru, 7, 31.

[7] Damasio, A.R. (1999) The Feeling of What Happens: Body and Emotions and the Making of Consciousness. Harcourt Brace, New York.

[8] Brenner, C. (2002) Conflict, Compromise Formation, and the Structural Theory. The Psychoanalytic Quarterly, 71, 407-408. https://doi.org/10.1002/j.2167-4086.2002.tb00519.x

[9] Fenichel, O. (1945) The Psychoanalytic Theory of Neurosis. W.W. Norton \& Company, Inc., New York.

[10] Kandel, E.R. (2012) The Age of Insight. Random House, Inc., New York, 297.

[11] Leucht, S., et al. (2017) Sixty Years of Placebo-Controlled Antipsychotic Drug Trials in Acute Schizophrenia: Systemic Review, Bayesian Meta-Analysis, and Meta Regression of Efficacy Predictors. American Journal of Psychiatry, 174, 927-942. https://doi.org/10.1176/appi.ajp.2017.16121358

[12] Kotov, R., et al. (2017) Declining Clinical Course of Psychotic Disorders over the Two Decades Following First Hospitalization: Evidence from the Suffolk County Mental Health Project. American Journal of Psychiatry, 174, 1064-1074. https://doi.org/10.1176/appi.ajp.2017.16101191

[13] American Psychiatric Association (2013) Diagnostic and Statistical Manual of Mental Disorders. 5th Edition, American Psychiatric Publishing Inc., Arlington.

[14] Arlow, J. (1991) Psychoanalysis: Clinical Theory and Practice. International Universities Press, Madison, 101.

\section{Abbreviations}

CNS-Central Nervous System

ANS-Autonomic Nervous System

PNS-Peripheral Nervous System

DSM-V-Diagnostic and Statistical Manual of Mental Disorders 